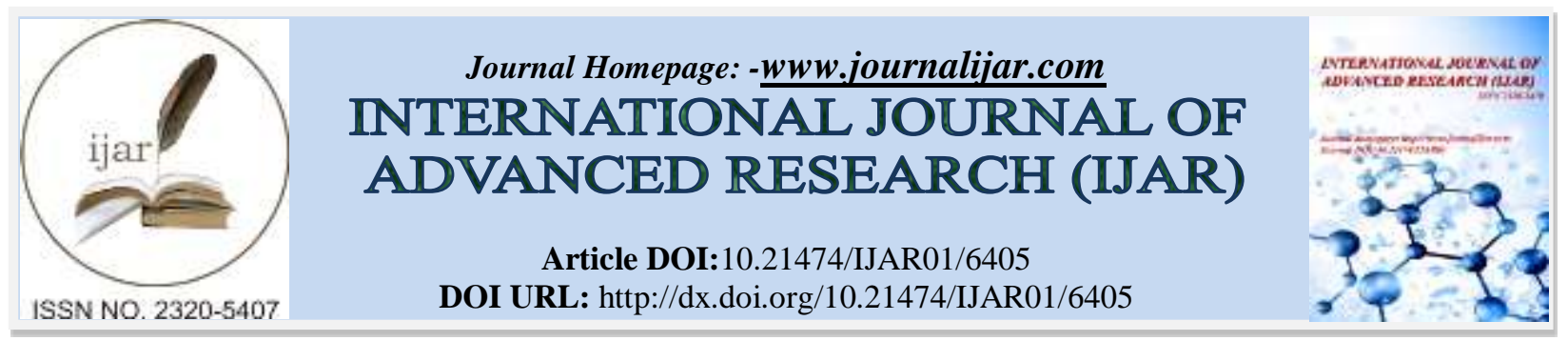

RESEARCH ARTICLE

\title{
SYSTEMIC ANTIBIOTIC PRESCRIPTION PATTERN IN NATIONAL GUARD OUTPATIENT CLINICS, JEDDAH, SAUDI ARABIA.
}

\author{
Abdulaziz Abdulnasir Aldahlawi, Samer Abdulmoghny Alamri, Ahmed Mohammed Alfaidi and Mohammed \\ Abdullah Babgi. \\ King Saud bin Abdulaziz University for Health Sciences - Jeddah, Saudi Arabia.
}

\section{Manuscript Info}

Manuscript History

Received: 01 December 2017

Final Accepted: 03 January 2018

Published: February 2018

Keywords:-

Antibiotics, MRSA, antibiotic misuse,

Antibiotic Resistance, Prescription

Antibacterial Agent, Pathogenic

Bacteria.

\begin{abstract}
Background and Aim: Antibiotics are one of the most prescribed medications worldwide. The misuse of antibiotics is an international health issue that has led to the emergence of antibiotics resistance. The inappropriate prescription of antibiotics plays a major role in the development of microbial resistance. The aim of this study is to evaluate the pattern and extent of antibiotic prescription in outpatient clinics, King Abdulaziz Medical City (KAMC), National Guard Hospital- Jeddah, Saudi Arabia.

Methods: A total of 5472 prescriptions were reviewed on average of 240 prescriptions per day from $28^{\text {th }}$ of January to $29^{\text {th }}$ of February 2016. Prescriptions containing systemic antibiotics were analyzed for the main elements of medication order. The World Health Organization (WHO) core drug use indicators were measured.

Results: The percentage of prescriptions that contained systemic antibiotics was $8.2 \%(\mathrm{n}=449)$ out of all prescriptions reviewed during the study period. Only $8 \%(n=36)$ of prescriptions contained more than one antibiotic agent, while the majority had single antibiotic. Family medicine clinics constituted 37\% $(\mathrm{n}=168)$ of all prescribed antibiotics. The diagnosis was missing in $8.5 \%(\mathrm{n}=38)$ of prescriptions. Allergy was not documented in $6.2 \%(\mathrm{n}=28)$ of prescriptions. Upper respiratory tract disease (URTI) was the most common diagnosis in $35.6 \%(\mathrm{n}=160)$ of patients who received antibiotics. The most frequently prescribed antibiotics were Augmentin and Azithromycin in $21.1 \%(\mathrm{n}=95)$ and $20.7 \%$ ( $\mathrm{n}=93$ ) of patients, respectively.

Conclusion: The percentage of systemic antibiotic prescriptions in KAMC outpatient clinics was acceptable according to the WHO indicators. However, the missing information in some of prescription forms necessitates further monitoring and education on the rationale of antibiotics prescription.

Copy Right, IJAR, 2018,. All rights reserved.
\end{abstract}

\section{Introduction:-}

Since the discovery of penicillin in 1920s, antibiotics continue to be cornerstone for the treatment of a wide variety of infections (1). The use of antibiotics can be empirical, definitive, or prophylactic therapy. The extensive use and misuse of antimicrobial agents led to the emergence of the global threat of antibiotic resistance, which is defined as 
"resistance of a microorganism to an antimicrobial drug that was originally effective for treatment of infections caused by it" (2). The total consumption of certain antibiotics is significantly linked to the development of resistance against these antibiotics (3). Upper respiratory tract infection (URTI) is the most common indication for antibiotic prescription, especially in the pediatric population. Azithromycin and Amoxicillin/Clavulanic Acid (Augmentin) are the most common antibiotics prescribed for URTI(4) A study done in the United States showed that Azithromycin was the most commonly prescribed antibiotic, also, family physicians were the most prescribing department of antibiotics (5). In outpatient clinics in Saudi Arabia, the percentage of prescriptions that included antibiotics was about $16 \%(6,7)$ and in another study, $15 \%$ of the prescriptions lacked the diagnosis (8). A study in Saudi Arabia revealed that Carbapenem- resistant acinetobacter baumannii (CRAB) has increased significantly during the past years. The organism's susceptibility in 2006 to Meropenem and Imipenem was $64-81.2 \%$ in comparison to data from 2012 showing a decrease of susceptibility to 8.3\%-11\% (10). A national surveillance study showed 32\% prevalence of methicillin-resistant staphylococcus aureus (MRSA) organisms. Moreover, 33\% and 26\% of streptococcus pneumoniae isolates were resistant to Penicillin G and Erythromycin respectively (11). The current study aims to evaluate the pattern and extent of antibiotic prescription in outpatient clinics of the National Guard Hospital and affiliated Primary Healthcare Centers in Jeddah, Saudi Arabia.

\section{Methods:-}

This is a descriptive single center retrospective chart review study of systemic antibiotic prescriptions in the outpatient clinics of the National Guard Hospital, Jeddah, Saudi Arabia. Prescriptions from January to February 2016 were reviewed for current systemic antibiotic use. A data collection was designed and included demographics, antibiotic prescription details, and the prescribing department. Details regarding antibiotic prescriptions included the name of the drug, route of administration, dose, duration, and the current diagnosis for which the drug was prescribed. Moreover, we checked for any other previous antibiotic use and documented allergies. We identified the most common type of antibiotics being dispensed, and the most frequent dispensing department. The World Health Organization (WHO) core drug use indicators were measured to assess the extent of antibiotic dispensing as the follow: The percentage of encounters with antibiotic prescribed $=$ (number of patient encounters with an antibiotic prescribed/the total number of encounters surveyed) $\times 100$.

\section{Statistical analysis:-}

Data was analyzed using IBM SPSS statistical software. Descriptive statistics were applied. Student's $t$ test was used to compare means of two independent groups. Chi square test was used for categorical variables. Non-parametric statistics were applied as appropriate. Level of significance used was $\mathrm{p}<0.05$.

\section{Results:-}

The total number of reviewed prescriptions was 5472, out of which $8.2 \%(\mathrm{n}=449)$ contained current systemic antibiotics and accordingly included in the study. The mean $( \pm$ SD) age of the studied sample was $38.29( \pm 21.25)$ years (range 1-101 years), with $41.9 \%(n=188)$ were between 30 to 60 years old. Only $8 \%(n=36)$ of the antibiotic prescriptions contained more than one antibiotic agent. Sixty seven percent $(n=301)$ of the patients had no recorded previous antibiotic prescription. The diagnosis and allergy status were not documented in $8.5 \%(\mathrm{n}=38)$ and $6.2 \%$ $(\mathrm{n}=28)$ of the prescriptions, respectively. Medication allergy was documented in 3.8\% $(\mathrm{n}=17)$ of prescriptions, while $0.7 \%(n=3)$ of patients were documented to have food allergy (Table 1$)$. The most frequently prescribed antibiotics were Augmentin, Azithromycin, Cefuroxime in 21.1\% ( $\mathrm{n}=95), 20.7 \% \quad(\mathrm{n}=93), 11.6 \% \quad(\mathrm{n}=52)$ of patients, respectively(Table 2). Upper respiratory tract infections (URTI) were the most common diagnosis in 35.6\% ( $\mathrm{n}=160)$ of patients who received antibiotics, followed by genitourinary diseases (GUD) with 16.3\% (n=73) (Figure 1). Among disease category, 25\% of URTI were treated by Augmentin. Moreover, Azithromycin was used to treat $33.1 \%$ of URTI cases. The most frequent drug used for the treatment of genitourinary diseases was ciprofloxacin with a percentage of $28.8 \%$. Sixty seven percent of antibiotics were prescribed for one week or less. Antibiotics for URTIs were prescribed for a duration of 7 days in $33.8 \%(n=54), 5$ days in $23.1 \%(n=37)$, and 3 days in $30.6 \%$ $(n=49)$. Few conditions were treated for longer duration, such as, cellulitis, diabetic foot ulcers, diverticulitis, inflammatory bowel disease, urethritis, pilonidal sinus, candidiasis, and pharyngitis. Family medicine was the highest prescribing department, accounting for $37 \%(\mathrm{n}=168)$ of all prescribed antibiotics. In addition, surgical subspecialties including; ENT, ophthalmology, orthopedics, urology, neurosurgery, and plastic surgery represent $20.5 \%(\mathrm{n}=92)$, followed by general medicine and general surgery with $11.6 \%(\mathrm{n}=52)$ and $10.5 \%(\mathrm{n}=47)$, respectively (figure 2). 
Table 1.

\begin{tabular}{|c|c|}
\hline Allergy Status & Antibiotic Prescription \% (n) \\
\hline No Known Allergy & $89.3 \%(401)$ \\
\hline Medication Allergy & $3.8 \%(17)$ \\
\hline Food Allergy & $0.7 \%(3)$ \\
\hline Not documented & $6.2 \%(28)$ \\
\hline
\end{tabular}

Table 2.

\begin{tabular}{|c|c|}
\hline & \\
\hline & $21.1(\mathrm{n}=95)$ \\
\hline Augmentin & $20.7(\mathrm{n}=93)$ \\
\hline Azithromycin & $11.5(\mathrm{n}=52)$ \\
\hline Cefuroxime & $9.7(\mathrm{n}=44)$ \\
\hline Ciprofloxacin & $8.4(\mathrm{n}=38)$ \\
\hline Amoxicillin & \\
\hline
\end{tabular}

Figure 1

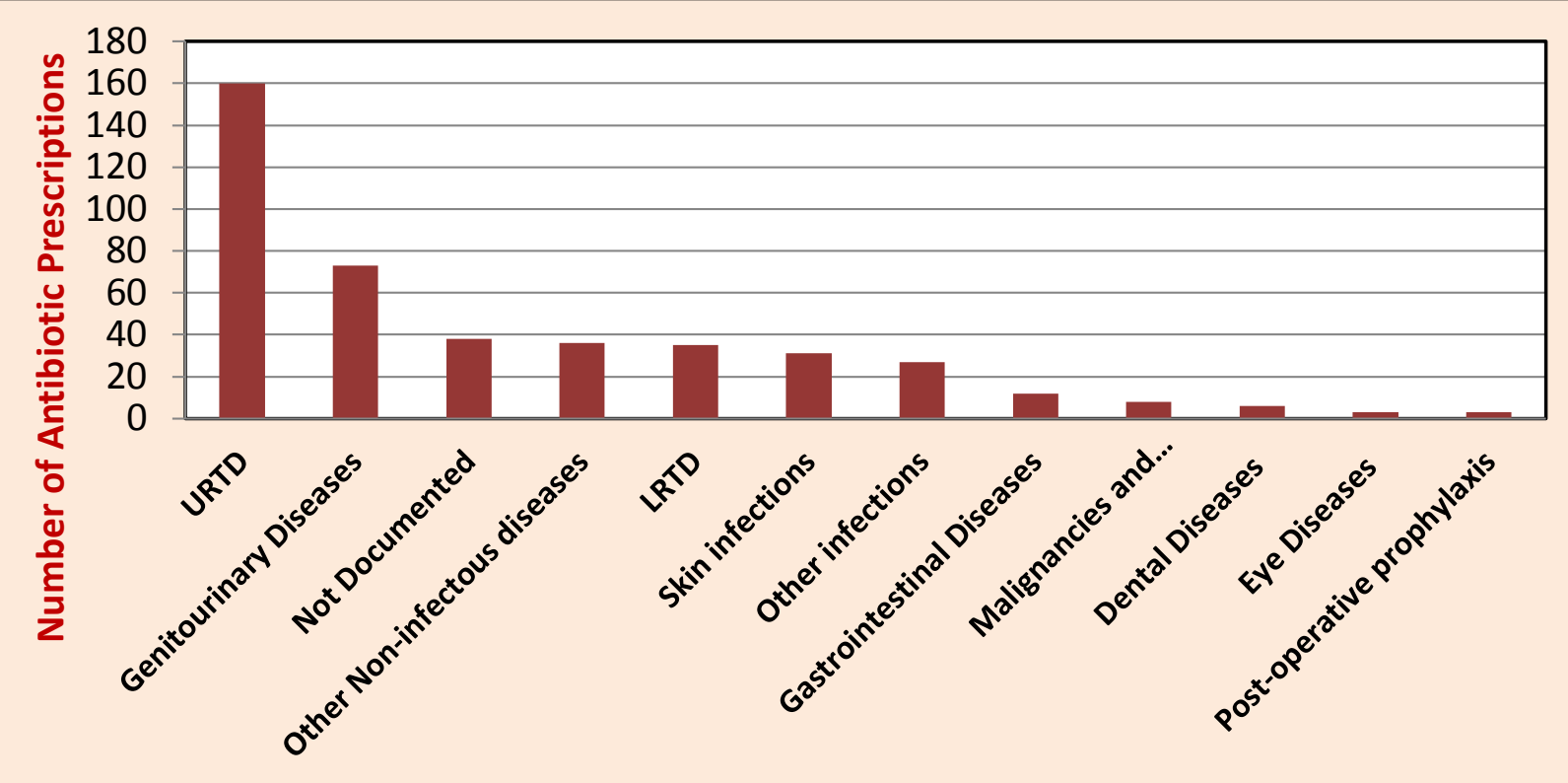

Diagnosis per Prescription 


\section{Figure 2.}

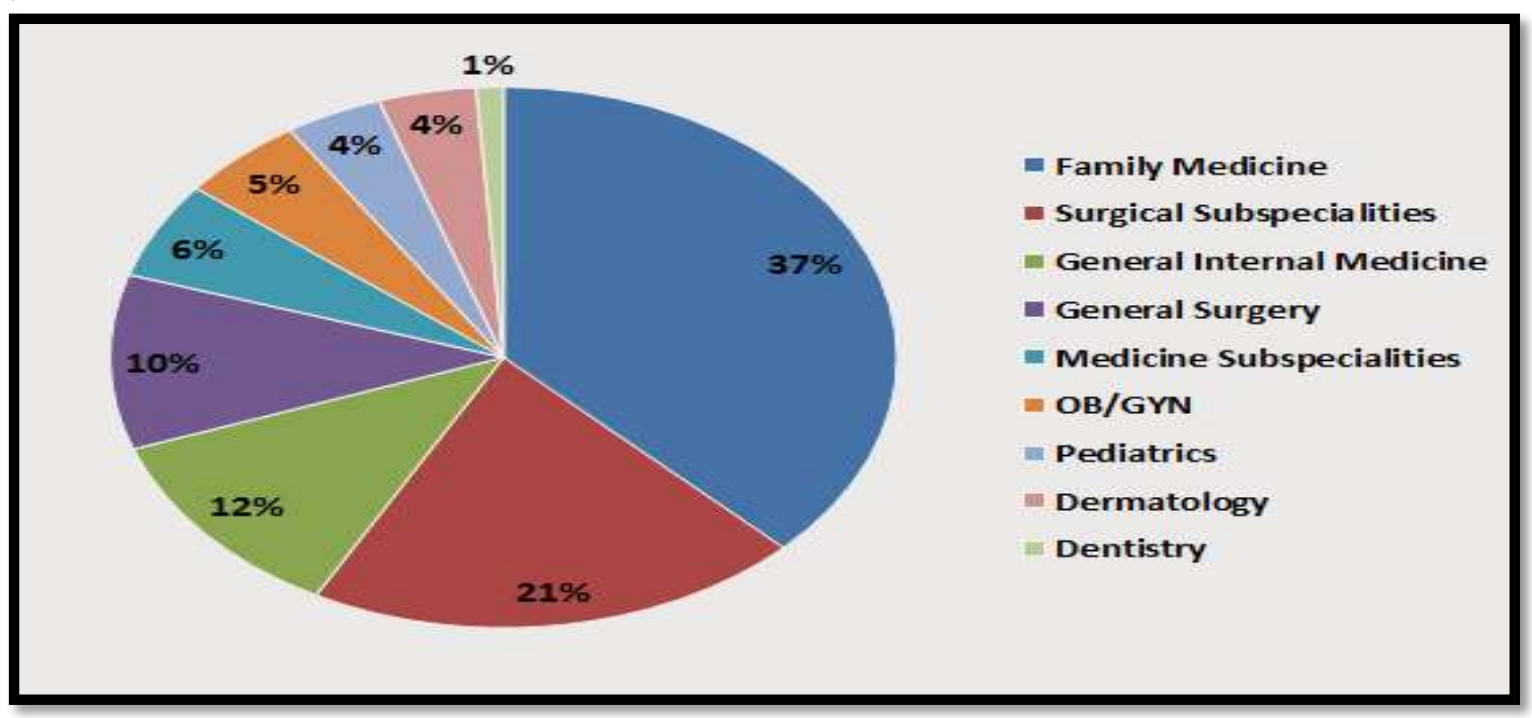

\section{Discussion:-}

According to $\mathrm{WHO}$ in 1993, prescription of antibiotics up to $25 \%$ of encounters is acceptable in countries where infectious diseases are common. Our data showed antibiotic prescription of $8.2 \%$, which is even lower than the WHO maximum requirement. In addition, more than one third of antibiotics were prescribed to treat URTIs, which are not necessarily treated by antibiotics, increasing the risk of antibiotic resistance. The main factors contributing to antibiotic resistance in Saudi Arabia include non-restricted and over prescription of antibiotics, easy access to these drugs in private pharmacies, inappropriate hand hygiene, and the yearly gathering of pilgrimage from around the world (9).

Case diagnosis and allergy were not reported in about $10 \%$ and 5\%, respectively of the prescriptions, which is considered as non-compliance with the hospital policy. Stating clinical diagnosis and allergy would help to avoid inappropriate drug dispense. According to an international study, drug allergy accounts for $10 \%$ of the whole adverse drug reactions (ADRs) (12). In our sample, 3.8\% ( $\mathrm{n}=17)$ had medications' allergy. However, considering that we reviewed only prescriptions, an accurate measurement was not feasible due to possible underestimation.

Family medicine department was the highest in prescribing antibiotics in our sample accounting for 37\% ( $\mathrm{n}=168$ ), which is relatively higher than other previous studies where family medicine department prescribed $24 \%$ of all antibiotic prescriptions (5). Antibiotics against URTIs and GUD were prescribed for a duration of one week or less. The duration to treat these diseases in general favors a shorter duration courses (13). A specified duration of antibiotics use against number of diseases has been well established in the literature. For instance, treatment of uncomplicated female urinary tract infections (UTI) is restricted to 3 days, while community acquired pneumonia for 5 days (13). Prolonged antibiotic use for a duration of4-6 weeks is advised in bacteremia by S.aureus, enterococcal endocarditis, osteomyelitis, or abdominal abscesses $(13,14)$. Establishing a defined period for use will help patients to avoid unfavorable events that could result from the inappropriate use of antibiotics. Unnecessarily prolonged use of antibiotics is associated with the rise of resistant organisms against antibiotics and adherence issues from patient side (13). In our sample, URTI was the most common indication for antibiotic prescription in 35.6\% $(\mathrm{n}=160)$. Reviewing the literature, URTIs are the leading indication for prescribing antibiotics in $41 \%$ of prescriptions. Furthermore, broad spectrum antibiotics constituted $83 \%$ of URTIs prescriptions in children $(4,15)$.

In regards to pattern of antibiotics prescription, our data is relatively close to the international pattern of outpatient oral antibiotics dispensing regimen. According to the annual report by the Centers for Disease Control and Prevention (CDC), the most commonly prescribed agents in the US (2014) in order were Amoxicillin, Azithromycin, Amoxicillin/Clavulanic Acid (Augmentin), Cephalexin, and Ciprofloxacin (5, 16). In comparison to our institute, the drugs in order were Amoxicillin/Clavulanic Acid (Augmentin), Azithromycin, Cefuroxime, Ciprofloxacin, and Amoxicillin. 
In conclusion, the percentage of systemic antibiotic prescriptions in the National Guard's outpatient clinics in Jeddah was acceptable according to the WHO indicators. However, the missing information in some of prescription forms necessitates further education on the rationale use of antibiotics, and efforts should be directed toward further assessment and monitoring for antibiotics use among healthcare professionals as well as the public. Multi-level approach targeting physicians, patients, and the public should be implemented to optimize the proper use of antibiotics.

\section{Acknowledgment:-}

We would like to extend our thanks and graduate to Dr. Mohammed Aseeri for his great efforts in facilitating the process of data collection and continuous support, and Dr. Khalid Mobarak Al-Shahrani

for providing access to the out-patient's pharmacy records

\section{References:-}

1. Aminov RI. A brief history of the antibiotic era: lessons learned and challenges for the future. Front Microbiol. 2010;1:134.

2. Goodman LS, Gilman A, Brunton LL. Goodman \& Gilman's manual of pharmacology and therapeutics. New York: McGraw-Hill Medical; 2008. ix, 1219 p. p.

3. Bergman M, Huikko S, Huovinen P, Paakkari P, Seppälä H, Renkonen R, et al. Macrolide and azithromycin use are linked to increased macrolide resistance in Streptococcus pneumoniae. Antimicrobial agents and chemotherapy. 2006;50(11):3646-50.

4. Al-Niemat SI, Aljbouri TM, Goussous LS, Efaishat RA, Salah RK. Antibiotic prescribing patterns in outpatient emergency clinics at Queen Rania Al Abdullah II Children's Hospital, Jordan, 2013. Oman medical journal. 2014;29(4):250.

5. Hicks LA, Bartoces MG, Roberts RM, Suda KJ, Hunkler RJ, Taylor TH, et al. US outpatient antibiotic prescribing variation according to geography, patient population, and provider specialty in 2011. Clinical Infectious Diseases. 2015:civ076.

6. Oqal M, Elmorsy S, Alfhmy A, Alhadhrami R, Ekram R, Althobaiti I, et al. Patterns of antibiotic prescriptions in the outpatient department and emergency room at a Tertiary Care Center in Saudi Arabia. Saudi Journal of Medicine and Medical Sciences. 2015;3(2):124-9.

7. Eldalo AS. Drug Prescribing Pattern among Physicians in an Outpatient Department of Tertiary Hospital, KSA. British Journal of Pharmaceutical Research. 2015;5(2):117.

8. Irshaid YM, Al-Homrany MA, Hamdi AA, Adjepon-Yamoah KK, Mahfouz AA. A pharmacoepidemiological study of prescription pattern in outpatient clinics in Southwestern Saudi Arabia. Saudi medical journal. 2004;25(12):1864-70.

9. Zowawi HM. Antimicrobial resistance in Saudi Arabia: An urgent call for an immediate action. Saudi Medical Journal. 2016;37(9):935-40.

10. Al-Obeid S, Jabri L, Al-Agamy M, Al-Omari A, Shibl A. Epidemiology of extensive drug resistant Acinetobacter baumannii (XDRAB) at Security Forces Hospital (SFH) in Kingdom of Saudi Arabia (KSA). Journal of Chemotherapy. 2015;27(3):156-62.

11. Shibl AM, Memish ZA, Kambal AM, Ohaly YA, Ishaq A, Senok AC, et al. National surveillance of antimicrobial resistance among Gram-positive bacteria in Saudi Arabia. Journal of Chemotherapy. 2014;26(1):13-8.

12. Thong BYH, Tan TC. Epidemiology and risk factors for drug allergy. British journal of clinical pharmacology. 2011;71(5):684-700.

13. Leekha S, Terrell CL, Edson RS. General Principles of Antimicrobial Therapy. Mayo Clinic Proceedings. 2011;86(2):156-67.

14. el Moussaoui R, de Borgie CAJM, van den Broek P, Hustinx WN, Bresser P, van den Berk GEL, et al. Effectiveness of discontinuing antibiotic treatment after three days versus eight days in mild to moderate-severe community acquired pneumonia: randomised, double blind study. BMJ : British Medical Journal. 2006;332(7554):1355-.

15. Shapiro DJ, Hicks LA, Pavia AT, Hersh AL. Antibiotic prescribing for adults in ambulatory care in the USA, 2007-09. Journal of Antimicrobial Chemotherapy. 2013:dkt301.

16. Control CfD, Prevention. Outpatient antibiotic prescriptions-United States, 2013. Available; 2016. p. 203. 